\title{
Spinal anesthesia for urgent Cesarean section in a patient with uncontrolled hyperthyroidism due to Graves' disease - A case report -
}

Received February 3, 2020

Revised April 7, 2020

Accepted May 25, 2020

\author{
Corresponding author \\ Jeongho Kim, M.D. \\ Department of Anesthesiology and \\ Pain Medicine, Dong-A University \\ College of Medicine, 26 \\ Daesingongwon-ro, Seo-gu, Busan \\ 49201, Korea \\ Tel: 82-51-240-5390 \\ Fax: 82-51-247-7819 \\ E-mail: surfy07@gmail.com
}

\section{Sangyoong Park, Soron Choi, Joonho Jeong, and Jeongho Kim}

Department of Anesthesiology and Pain Medicine, Dong-A University College of Medicine, Busan, Korea

\begin{abstract}
Background: Effective treatment of Graves' disease during pregnancy is important because uncontrolled hyperthyroidism is associated with increased fetal and maternal morbidity and mortality. While there have been case reports of patients with Graves' disease who failed to achieve euthyroid state during pregnancy, anesthesiologists rarely encounter patients with uncontrolled hyperthyroidism undergoing urgent Cesarean section.
\end{abstract}

Case: A 31-year-old pregnant patient had uncontrolled hyperthyroidism due to Graves' disease despite medical treatment. Her signs and symptoms suggested fetal distress and aggravation of the disease, leading to hospitalization. After a failed induction for vaginal delivery, an urgent Cesarean section was performed under spinal anesthesia via an interlaminar approach using $9 \mathrm{mg}$ of $0.5 \%$ heavy bupivacaine and $20 \mu \mathrm{g}$ of fentanyl. It resulted in successful delivery, with no perioperative complications for the mother and neonate.

Conclusions: This case demonstrates that spinal anesthesia may provide clinical stability to patients with uncontrolled hyperthyroidism undergoing urgent Cesarean section.

Keywords: Cesarean section; Emergencies; Hyperthyroidism; Spinal anesthesia.
Hyperthyroidism is defined as an excessive production and release of thyroid hormone by the thyroid gland, resulting in inappropriately high serum levels and an accelerated metabolic state [1]. Hyperthyroidism in women of childbearing age is most commonly due to Graves' disease (GD), which occurs in $0.4-1.0 \%$ of women before pregnancy and in approximately $0.2 \%$ of women during pregnancy [2]. Given the complexity surrounding thyroid physiology and thyroid illness during pregnancy, the effective treatment of GD during pregnancy is challenging, but is vital for the health of the mother and the fetus [2]. Poor control of hyperthyroidism is associated with pregnancy loss, pregnancy-induced hypertension, prematurity, low birth weight, intrauterine growth restriction, still birth, thyroid storm, and maternal congestive heart failure [2].

We describe the case of a patient who failed to achieve euthyroid state despite perinatal care for GD and underwent urgent Cesarean section (C-section) with spinal anesthesia for delivery.

\section{CASE REPORT}

Informed consent was obtained from the patient for the publication of this report.

A 31-year-old patient with a gestational age (GA) of 16 weeks and 6 days visited the Obstetrics-Gynecology outpa-

This is an Open Access article distributed under the terms of the Creative Commons Attribution Non-Commercial License (http://creativecommons.org/licenses/by-nc/4.0) which permits unrestricted non-commercial use, distribution, and reproduction in any medium, provided the original work is properly cited.

Copyright (c) the Korean Society of Anesthesiologists, 2020 
tient clinic at our hospital due to uncontrolled hyperthyroidism. Thyroid function tests (TFTs) revealed a thyroid-stimulating hormone (TSH) level of $<0.04 \mu \mathrm{lU} / \mathrm{ml}$ (normal range: $0.3-4.1 \mu \mathrm{lU} / \mathrm{ml}$ ), a free thyroxine (FT4) level of $10.14 \mathrm{ng} / \mathrm{dl}$ (normal range: $0.78-2.0 \mathrm{ng} / \mathrm{dl}$ ), and a TSH receptor antibody (TRAb) level of 107.4 IU/L (normal range: $0-14 \mathrm{IU} / \mathrm{L}$ ). The patient had become pregnant in 2018 while taking methimazole (MMI) for GD, which was diagnosed in 2012. After she became pregnant, the medication was switched to propylthiouracil (PTU), but severe emesis gravidarum interfered with proper ingestion. After visiting our clinic, the patient underwent a series of laboratory tests while taking MMI at 20 mg daily (Table 1), which was increased to $40 \mathrm{mg}$ daily at GA 22 weeks and 2 days due to a consistently high FT4 level. However, despite the increased dosage, the TFTs remained abnormal and the patient complained of palpitation and anxiety, resulting in a second dose increase to $60 \mathrm{mg}$ of MMI daily and the addition of $6.25 \mathrm{mg}$ of carvedilol at GA 28 weeks and 2 days. Subsequently, she maintained a FT4 level of $<3.0 \mathrm{ng} / \mathrm{dl}$ (normal range: 0.93-1.7 ng/dl), systolic blood pressure (BP) of $<150 \mathrm{mmHg}$, and heart rate (HR) of $<100$ beats/min at follow-up examinations. At GA 35 weeks, decreased fetal movements were observed and her systolic BP increased and remained at $>150 \mathrm{mmHg}$ with complaints of palpitation, anxiety and depressive mood, which led to her hospi- talization. The patient also showed minimal variability during prenatal nonstress testing and was not reactive to fetal acoustic stimulation tests while taking oral MMI (60 mg daily), propranolol (120 mg daily), and 3 drops of Lugol's solution (LS) three times daily. At GA 35 weeks and 6 days, the patient still complained of the above-mentioned symptoms and had generalized edema on examination. Subsequently, labor induction for vaginal delivery was commenced; however, the induction attempt failed and an emergency C-section was scheduled for non-reassuring fetal status based on the results of a nonstress test showing persistently minimal fetal heart rate variability. Blood test results showed a TSH level of $<0.005 \mu \mathrm{IU} / \mathrm{ml}$, triiodothyronine (T3) level of $2.18 \mathrm{ng} / \mathrm{ml}$ (normal range: 0.8-2.0 ng/ $\mathrm{ml}$ ), FT4 level of $3.18 \mathrm{ng} / \mathrm{dl}$, and TRAb level of $55.0 \mathrm{IU} / \mathrm{L}$ (normal range: 0-14 IU/L). There were no abnormal findings on chest radiography or transthoracic echocardiography; electrocardiogram (ECG) showed sinus tachycardia.

On the day of surgery, the patient was orally administered $20 \mathrm{mg}$ of MMI, 3 drops of LS, and $40 \mathrm{mg}$ of propranolol preoperatively. She was also intravenously administered $2 \mathrm{mg}$ of dexamethasone and a nasogastric (NG) tube was inserted for further administration of anti-thyroid agents. Since the patient refused premedication or anxiolytics, a preoperative consultation was done to reduce the patient's anxiety; this involved detailed explanations about the an-

Table 1. Results of Serial Thyroid Function Tests

\begin{tabular}{|c|c|c|c|c|}
\hline Gestational age & $\mathrm{TSH}(\mu \mathrm{lU} / \mathrm{ml})$ & FT4 (ng/dl) & T3 (ng/ml) & Progress note \\
\hline 18 wk and $6 \mathrm{~d}$ & $<0.005$ & 6.59 & 5.93 & \\
\hline 22 wk and $2 d$ & & 6.49 & $>6.51$ & MMl dose was escalated from $20 \mathrm{mg}$ to $40 \mathrm{mg}$ \\
\hline 25 wk and $1 \mathrm{~d}$ & & 4.24 & 3.05 & \\
\hline \multirow[t]{2}{*}{28 wk and $2 \mathrm{~d}$} & & $>7.77$ & 5.70 & MMl dose was escalated from $40 \mathrm{mg}$ to $60 \mathrm{mg}$ \\
\hline & & & & 6.25 mg of Carvedilol was newly prescribed \\
\hline $32 \mathrm{wk}$ and $2 \mathrm{~d}$ & $<0.005$ & 2.80 & 3.75 & \\
\hline $34 \mathrm{wk}$ and $0 \mathrm{~d}$ & $<0.005$ & 3.84 & 5.11 & \\
\hline 34 wk and $2 d$ & $<0.005$ & 3.49 & 3.84 & MMI 60 mg, Carvedilol 6.25 mg \\
\hline 35 wk and $5 d$ & & 3.18 & 2.42 & $\begin{array}{l}\text { MMI } 60 \text { mg, Propranolol } 120 \text { mg, Lugol solution } 9 \\
\text { drops, Dexamethasone } 6 \text { mg }\end{array}$ \\
\hline \multirow[t]{2}{*}{35 wk and $6 \mathrm{~d}$} & & 2.80 & 2.18 & The day of the surgery \\
\hline & & & & Results are preoperative \\
\hline POD 0 & & 3.03 & 1.82 & Results are postoperative \\
\hline POD 1 & & 2.57 & 1.88 & $\begin{array}{l}\text { MMI } 60 \text { mg, Propranolol } 120 \text { mg, Lugol solution } 9 \\
\text { drops, Dexamethasone } 6 \text { mg }\end{array}$ \\
\hline POD 2 & & 2.18 & 1.85 & \\
\hline POD 3 & & 2.17 & 1.75 & MMI 30 mg, Propranolol 80 mg \\
\hline \multirow[t]{2}{*}{ POD 14} & $<0.005$ & 2.43 & 3.38 & $\mathrm{MMI}$ dose was escalated from $30 \mathrm{mg}$ to $40 \mathrm{mg}$ \\
\hline & & & & Propranolol $80 \mathrm{mg}$ \\
\hline
\end{tabular}

TSH: thyroid stimulating hormone (normal range, 0.27-5.2 $\mu \mathrm{lU} / \mathrm{ml}$ ), FT4: free thyroxine (normal range, 0.93-1.7 ng/dl), T3: triiodothyronine (normal range, $0.8-2.0 \mathrm{ng} / \mathrm{ml}$ ), MMl: methimazole, POD: postoperative day. 
esthetic and surgical methods. Subsequently, the patient was transferred to the operating room with prepared MMI and PTU in powder form and LS. Basic monitoring, including BP, HR, ECG, and pulse oximetry was applied. The initial vital signs were as follows: BP 136/76 mmHg, HR 97 beats/min, oxygen saturation $\left(\mathrm{SpO}_{2}\right) 95 \%$, and body temperature $37.0^{\circ} \mathrm{C}$.

After verifying the patient's information, arterial and central venous catheters were inserted. While maintaining the right lateral decubitus position, spinal anesthesia was performed via an interlaminar approach at the L4-5 level. Cerebrospinal fluid was checked, after which $9 \mathrm{mg}$ of $0.5 \%$ heavy bupivacaine and $20 \mu \mathrm{g}$ of fentanyl were injected into the subarachnoid space. Approximately 5 minutes later, a sensory block up to the T6 level was confirmed and a C-section was successfully performed, resulting in the delivery of a female baby who weighed $2.7 \mathrm{~kg}$ at birth with Apgar score of 9 and 10 at 1 and 5 min, respectively. Time between skin incision and delivery was about $3 \mathrm{~min}$ and the total operation time was $30 \mathrm{~min}$. Anxiolytics were not used throughout the surgery according to the patient's wish. The patient's vital signs before spinal anesthesia administration were maintained as follows: BPs of 140162/69-95 mmHg, HRs of 85-103 beats/min, and $\mathrm{SpO}_{2} \mathrm{~s}$ of 96-100\%. From the start of anesthesia until completion of the operation, the vital signs were maintained at BPs of 98131/50-69 mmHg, HRs of 92-95 beats/min, and $\mathrm{SpO}_{2}$ of $100 \%$ (Fig. 1). Blood loss was $300 \mathrm{ml}$, urine output was 350 $\mathrm{ml}$, and 2,000 $\mathrm{ml}$ of crystalloid solution was administered intravenously during the procedure. The patient was transferred to the post-anesthesia care unit, where she stayed for 20 min without complications; she was subsequently transferred to the general ward.

For postoperative pain control, intravenous patient-controlled analgesia (IVPCA) was applied after surgery and the patient needed no other rescue analgesia. The IVPCA was prepared at a total volume of $100 \mathrm{ml}$ by mixing fentanyl 30 $\mu \mathrm{g} / \mathrm{kg}$ and ramosetron $0.6 \mathrm{mg}$ in normal saline, of which the baseline infusion rate, bolus demand dose, and lockout time were $1 \mathrm{ml} / \mathrm{h}, 1 \mathrm{ml}$, and $10 \mathrm{~min}$, respectively. Meanwhile, MMI (60 mg daily), Propranolol (120 mg daily), 3 drops of LS (thrice daily), and dexamethasone (6 mg daily) were administered up to postoperative day (POD) 2 . From POD 3, the dosage of MMI and propranolol was decreased to $30 \mathrm{mg}$ and $80 \mathrm{mg}$ daily, respectively. In the laboratory test results, TFT showed improvement in T3 to 1.75 ng/ml (normal: 0.8-2.0 ng/ml) and FT4 to $2.17 \mathrm{ng} / \mathrm{dl}$ (normal: $0.93-1.7 \mathrm{ng} / \mathrm{dl}$ ). The patient was discharged on POD 4 without any perioperative complication. The neonate also underwent a series of evaluations, due to prematurity and maternal Graves' disease, including TFT, skull X-ray, kidney ultrasonography, spinal cord ultrasonography, neurosonography, and echocardiogram. TFT revealed that the neonate had a euthyroid state and the other fore mentioned tests showed no abnormal findings.

\section{DISCUSSION}

Gestational transient thyrotoxicosis (GTT) is the most common cause of thyrotoxicosis during pregnancy, affecting 2-3\% of the European pregnant population. The FT4 level in GTT patients usually normalizes at a GA of 14-18

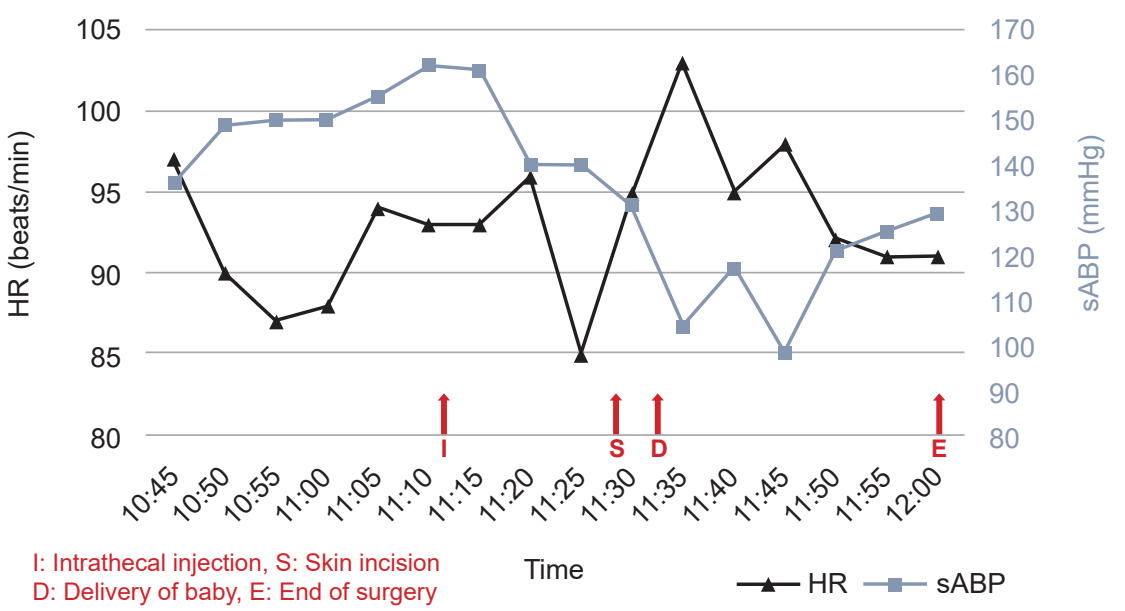

Fig. 1. Perioperative systolic arterial blood pressure (SABP) and heart rate (HR). The patient's SABP and HR before and after intrathecal injection are shown. Note that the SABP remained stable, and even decreased following the intrathecal injection. HR remained relatively stable throughout the procedure. This figure was prepared using Microsoft PowerPoint and PDF-Xchange Viewer. 
weeks, and a short-term follow-up observation period without anti-thyroid agent usage is recommended. When evaluating these patients, clinicians should carefully consider the differential diagnosis of hyperthyroidism in pregnancy. In such cases, GD can be differentiated from GTT based on a positive test result for TRAb [3]. In the present case, the patient was determined to have hyperthyroidism caused by GD because she was being treated for diagnosed GD prior to her pregnancy and her TRAb level was 107.4 IU/L (normal range: 0-14 IU/L) at the time of her first visit to the hospital.

Due to rare congenital anomalies that may occur from maternal use of MMI during embryogenesis [3], it is recommended that PTU should be used during the first trimester and that patients should subsequently be switched to MMI during the second trimester. The patient in the present case had uncontrolled hyperthyroidism due to hyperemesis gravidarum on her first visit to our hospital at GA 16 weeks and 6 days. The patient reported that she had been unable to properly eat and ingest her medications because of vomiting, resulting in weight loss of $5 \mathrm{~kg}$ in 3 weeks. Fortunately, from that point onwards, nausea and vomiting improved without any specific treatment. She was prescribed $20 \mathrm{mg}$ of MMI daily by the endocrinologist at our hospital, which was increased due to persistent hyperthyroidism in serial laboratory testing; however, despite the increased dose, the patient experienced only a partial response to treatment and a euthyroid state could not be reached. Linardi et al. [4] reported a case of a patient with resistant thyrotoxicosis, in which drug malabsorption, antidrug antibodies, rapid drug metabolism, and impaired intrathyroidal drug accumulation and action were suggested as possible mechanisms. In that case, thyrotoxicosis was well-controlled with $30 \mathrm{mg}$ of a corticosteroid (prednisolone) and the maximum dose of carbimazole [4]. In the present case, the patient was administered $6 \mathrm{mg}$ of dexamethasone intravenously to limit the peripheral conversion of thyroid hormone. This was in addition to the following oral medications prescribed by the endocrinologist: MMI for inhibiting thyroid hormone synthesis; beta-blocker for controlling hypermetabolic symptoms and blocking the peripheral conversion of inactive $\mathrm{T} 4$ to the active form of T3; and LS for blocking the release of stored thyroid hormone from the thyroid gland $[1,2]$.

When uncontrolled hyperthyroidism persists, it may be severely exacerbated by surgery or by the disease itself. Such exacerbations are referred to as "thyroid storm," which can be life-threatening. A thyroid storm is characterized by hyperpyrexia, tachycardia, and striking alterations in consciousness, and it cannot currently be diagnosed by laboratory testing [5]. In such cases, anti-thyroid agents are generally administered orally, but they may also be administered rectally [6]. There are even case reports of intravenous administration of MMI [7]. Since oral administration was the only option at our hospital, MMI, LS, and steroids were prepared for oral administration through the NG tube during the surgery. Meanwhile, the arterial catheter and central venous catheter were inserted for continuous arterial blood pressure monitoring and management in case of thyroid storm, which may require hemodynamic monitoring, aggressive volume resuscitation and medical treatment with multimodal approach $[1,8]$.

Anesthetic management of hyperthyroidism should focus on perioperative control of sympathetic stimulation, so that cardiovascular side effects are not manifested. Additionally, early detection of thyroid storm should also be taken into consideration due to the possibility of rapid deterioration within days or hours [8]. General anesthesia is often selected as the anesthetic method for patients with uncontrolled thyrotoxicosis requiring urgent surgery because it provides adequate sedation and minimal fluctuations of hemodynamic parameters. Moreover, propofol and remifentanil are believed to provide benefits to patients with thyrotoxicosis by lowering their BPs and HRs $[9,10]$. However, when performing general anesthesia, irritation caused by the endotracheal tube during induction and emergence may result in a dramatic sympathetic stimulation and resultantly increases in HR and BP. Furthermore, even during the maintenance phase, surgical manipulations with insufficient depth of anesthesia may also stimulate the sympathetic nervous systems of patients [11].

Neuraxial anesthesia may be a suitable alternative for patients with uncontrolled thyrotoxicosis since it can lower BP and HR through a sympathetic block, reduce adverse effects caused by histamine secreted in response to neuromuscular blocking agent administration, and provide adequate postoperative pain control. Moreover, considering that symptoms are an important component in the diagnosis of thyroid storm, maintaining consciousness has an advantage for early detection. However, cases involving the onset of thyroid storm before induction have been reported [12]. Additionally, psychological factors in the patient, including anxiety and agitation during the processes of applying the neuraxial anesthesia and performing the sur- 
gery, may lead to a thyroid storm. We chose neuraxial anesthesia as the anesthetic method after consultation with an obstetrician and with the expectation that pain control and changes in hemodynamic parameters would be beneficial to the patient. Accordingly, we obtained patient consent after providing sufficient explanation regarding anesthesia.

Considering that surgeries and severe emotional stress are known precipitating events of thyrotoxic storm, shortening the time required for anesthesia and surgery was thought to be important to prevent such a catastrophic event $[11,12]$. In this respect, epidural anesthesia was not considered as an optimal method because of its slower onset of blockade and failure rate compared to spinal anesthesia or combined spinal-epidural anesthesia (CSE) [13]. Additionally, spinal anesthesia could be a better alternative to CSE due to the shorter time needed to perform the procedure, although it is doubtful whether the statistical difference reported in various studies would be clinically meaningful [14]. On the other hand, postoperative analgesia was another important consideration. A systematic review that compared epidural analgesia (EA) with IVPCA for postoperative analgesia following intra-abdominal surgery in adults reported a small improvement in pain scores in the postoperative phase with an increased rate of failure to establish the allocated technique successfully when using EA [15].

For the following reasons, we chose spinal anesthesia for the anesthetic method. The patient had elevated BPs during induction, but stable BPs and HRs were observed from the confirmation of the sensory block to the completion of the surgery (Fig. 1). Moreover, there were no significant complications in either the patient or neonate before discharge

In conclusion, the anesthetic management of the urgent C-section, can be complicated by uncontrolled hyperthyroidism despite aggressive medical treatment. Our case demonstrates that spinal anesthesia could be a proper anesthetic method in such cases and may provide clinical stability.

\section{CONFLICTS OF INTEREST}

No potential conflict of interest relevant to this article was reported.

\section{AUTHOR CONTRIBUTIONS}

Conceptualization: Sangyoong Park. Supervision: Soron Choi. Writing-original draft: Joonho Jeong. Writing-review \& editing: Jeongho Kim.

\section{ORCID}

Sangyoong Park, https://orcid.org/0000-0001-7495-8025

Soron Choi, https://orcid.org/0000-0002-4173-8939

Joonho Jeong, https://orcid.org/0000-0001-7586-5592

Jeongho Kim, https://orcid.org/0000-0003-4447-2838

\section{REFERENCES}

1. Devereaux D, Tewelde SZ. Hyperthyroidism and thyrotoxicosis. Emerg Med Clin North Am 2014; 32: 277-92.

2. Alexander EK, Pearce EN, Brent GA, Brown RS, Chen H, Dosiou C, et al. 2017 Guidelines of the American Thyroid Association for the diagnosis and management of thyroid disease during pregnancy and the postpartum. Thyroid 2017; 27: 315 89.

3. Labadzhyan A, Brent GA, Hershman JM, Leung AM. Thyrotoxicosis of pregnancy. J Clin Transl Endocrinol 2014; 1: 140-4.

4. Linardi A, Michou E, Ilias I, Petychaki F, Kakoulidis I, Pappa A, et al. Resistant thyrotoxicosis due to Graves' disease in pregnancy: case report and review of the literature. Cureus 2018; 10: e3232.

5. Fleisher LA, Mythen M. Anesthetic implications of concurrent diseases. In: Miller's anesthesia. 8th ed. Edited by Miller RD, Cohen NL, Eriksson LI, Fleisher LA, Wiener-Kronish JP, Young WL: Philadelphia, Saunders/Elsevier. 2015, pp 1156-225.

6. Cooper DS. Antithyroid drugs. N Engl J Med 2005; 352: 905-17.

7. Hodak SP, Huang C, Clarke D, Burman KD, Jonklaas J, Janicic-Kharic N. Intravenous methimazole in the treatment of refractory hyperthyroidism. Thyroid 2006; 16: 691-5.

8. Satoh T, Isozaki O, Suzuki A, Wakino S, Iburi T, Tsuboi K, et al 2016 Guidelines for the management of thyroid storm from The Japan Thyroid Association and Japan Endocrine Society (First edition). Endocr J 2016; 63: 1025-64.

9. Matsumoto S, Shingu C, Hidaka S, Goto K, Hagiwara S, Iwasaka $\mathrm{H}$, et al. Anesthetic management of a patient with hyperthyroidism due to hydatidiform mole. J Anesth 2009; 23: 594-6.

10. Hwang W, Im D, Kim E. Persistent perioperative tachycardia and hypertension diagnosed as thyroid storm induced by a hydatidiform mole: a case report. Korean J Anesthesiol 2014; 67: 205-8.

11. Liao Z, Xiong Y, Luo L. Low-dose spinal-epidural anesthesia for 
Cesarean section in a parturient with uncontrolled hyperthyroidism and thyrotoxic heart disease. J Anesth 2016; 30: 731-4.

12. Hirvonen EA, Niskanen LK, Niskanen MM. Thyroid storm prior to induction of anaesthesia. Anaesthesia 2004; 59: 1020-2.

13. Kang HS. Regional anesthesia for cesarean delivery. Anesth Pain Med 2008; 3: 1-6.

14. Simmons SW, Dennis AT, Cyna AM, Richardson MG, Bright
MR. Combined spinal-epidural versus spinal anaesthesia for caesarean section. Cochrane Database Syst Rev 2019; 10: CD008100.

15. Salicath JH, Yeoh EC, Bennett MH. Epidural analgesia versus patient-controlled intravenous analgesia for pain following intra-abdominal surgery in adults. Cochrane Database Syst Rev 2018; 8: CD010434. 